Pacific Journal of Mathematics

MRANT MERS AND ANALTTC ACTR 


\section{INVARIANT MEANS AND ANALYTIC ACTIONS}

\section{TheOdoRE MitcheLL}

Let $T \times D \rightarrow D$ be a separately continuous analytic action of a semitopological semigroup $T$ on $D$, the open unit disk in the complex plane, and let $K$ be a compact $T$-invariant subset of $D$. The chief result of this paper is that if $A P(T)$, the space of almost periodic functions on $T$, has a left invariant mean, then $D$ contains a common fixed-point of $T$. As a special case, we show that a finite group of analytic self maps of $D$ has a common fixed-point in $D$.

M. M. Day [1] pioneered the investigation of the relationship between fixed-point properties of affine actions of a semigroup $T$ to the existence of an invariant mean on $T$.

A. T. Lau [9] obtained the result that if $A P(T)$ has a left invariant mean, then every equicontinuous affine separately continuous action $T \times K \rightarrow K$ of a semitopological semigroup $T$ on a compact convex subset $K$ of a locally convex (separated) linear topological space has a common fixed-point in $K$. (A converse to this result was also obtained in [9].) Variants of this result concerning the fixed-point properties of actions of semitopological semigroups $T$, when $A P(T)$ has a left invariant mean, were studied later by J. C. S. Wong [14] and by H. D. Junghenn [7]. The results alluded to in this paragraph are united by a common theme in their proofs; they were shown essentially by "pulling back" various spaces of real valued functions on $K$ into the space $A P(T)$.

However, there are certain fixed-point theorems about actions of $T$, when $A P(T)$ has a left invariant mean, which are not obtained by pull-back arguments alone, but which rely on some special structure theorem of the space on which $T$ acts. As an example, in [10] Lau showed that if $T \times I \rightarrow I$ is an equicontinuous action of $T$ on $I$, the closed unit interval of the real line, then $I$ contains a common fixed-point of $T$. But the proof of this makes use of a fixed-point theorem of T. Mitchell [11, Theorem 2, p. 149], which in turn rests on the fact that a compact group of continuous self-maps of $I$ has a common fixed-point in $I$ (see [5, 3.24, p. 333]). However, this last fact no longer holds if $I$ is replaced by an arbitrary disk $K$ in $n$ space, even if the group is required to be finite and Abelian. (For counter-examples, see R. Oliver [12, Theorem 7, p. 174].) Moreover, the result of Lau in [10] no longer holds if $I$ is replaced by such an arbitrary $K$.

Let $f, g$ be two continuous commuting self-maps of $\bar{D}$, the closed unit disk in the complex plane, and let $f, g$ be analytic on $D$. 
Shields [13] has shown that $f$ and $g$ have a common fixed-point in $\bar{D}$. We would like to generalize Shields' result to a statement of the following type:

${ }^{*}$ ) Let $T$ be a semitopological semigroup, and let $T \times \bar{D} \rightarrow \bar{D}$ be a separately continuous action for which each $t \in T$ is analytic on $D$. If $A P(T)$ has a left invariant mean, then $T$ has a common fixed-point in $\bar{D}$.

Unfortunately, the statement $\left(^{*}\right)$ is false, as the counterexample to Theorem 1 which is given after Corollary 2 will show. We shamelessly rectify this by imposing a new condition in the hypothesis of $\left(^{*}\right)$; that there exists a $T$-invariant compact subset of $D$. With this new condition, we can now drop the requirement that each $t \in T$ is defined, let alone continuous, on $\bar{D}-D$. However, the modified and now correct statement (Theorem 2 in Section 4) is not proved by pull-back arguments alone, but makes use of a special case, viz: A compact group of analytic self-maps of $D$ has a common fixed-point in $D$. Since, unlike the case of $[5,3.24$, p. 333], we could not find any such result in the literature, we were forced to prove this special case (Lemma 2 in $\S 3$ ) before we could obtain our other results.

2. Preliminaries. A semigroup is a set equipped with an associative binary product called the semigroup product. A topological semigroup is a semigroup with a Hausdorff topology in which the product st is jointly continuous. A topological group is a group having a Hausdorff topology for which the product $s t^{-1}$ is jointly continuous.

Let $C$ denote the complex plane, and let $D$ be the open unit disk $D=\{z \in C ;|z|<1\}$. If $A \subseteq C$, then Int $(A)$ denotes the set of all interior points of $A$. The space of all analytic maps $f: D \rightarrow C$ is designated by $E(D)$, where $E(D)$ has the (metric) topology of uniform convergence on compact subsets of $D$. The subspace of all analytic self-maps of $D$ is designated by $H(D)$. When $H(D)$ is given the semigroup product of functional composition, it becomes a topological semigroup in the topology of $E(D)$ [13, Lemma 1, p. 703]. If $S \subseteq E(D)$, then $\bar{S}$ denotes the closure of $S$ in $E(D)$.

A set $F \subseteq C$ for which there exist $w \in C$ and $\alpha>0$ such that $F=\{z \in C ;|w-z| \leqq \alpha\}$, is called a closed disk. Let $K \cong D$, where $K$ is compact. Then $\mathscr{J}(K)$ is the family of sets $\mathscr{J}(K)=\{F \subseteq D$; $K \subseteq F$ and $F$ is a closed disk $\}$. The set $C I R(K)=\cap\{F ; F \in \mathscr{J}(K)\}$ is called the circular hull of $K$. The set $K$ is said to be circular hulled if $K=C I R(K)$. If $S K \subseteq K$, where $S \subseteq E(D)$, then $K$ is called invariant under $S$. 


\section{Fixed-points.}

LEMMA 1. Let $K \subseteq D$, where $K$ is compact.

(a) $K$ is circular hulled iff $K$ is the intersection of a family of closed disks in $D$.

(b) $\operatorname{CIR}(K)$ is circular hulled.

(c) $K$ is circular hulled iff $K$ is the intersection of a family of circular hulled sets in $S$.

Proof. (a) If $K$ is circular hulled, then $K$ is the intersection of the family of closed disks $\mathscr{J}(K)$.

Now let $K=\cap\left\{F_{t} ; t \in I\right\}$, where $F_{t}$ is a closed disk in $D$ for each $t \in I$, an index set. Then $K \leqq F_{t}$, hence $F_{t} \in \mathscr{J}(K)$ for each $t \in I$. Thus we get

$$
C I R(K)=\bigcap\{F: F \in \mathscr{J}(K)\} \subseteq \cap\left\{F_{t} ; t \in I\right\}=K \cong C I R(K),
$$

which shows (a).

(b), (c). These follow immediately from (a).

LEMMA 2. Let $G \subseteq H(D)$, where $G$ is a compact topological group. Then $D$ contains a common fixed-point of $G$. If, in addition, $K$ is a compact $G$-invariant subset of $D$, then $C I R(K)$ contains a common fixed-point of $G$.

Proof. Since the semigroup product on $H(D)$ is jointly continuous, so is the map $H(D) \times D \rightarrow D$ given by $(f, z) \rightarrow f(z)$ for $f \in H(D)$, $z \in D$. (To see this, identify each $z \in D$ with the constant $z$ function on $D$.) For each $z \in D$, it follows by compactness of $G$ that $G(z)$ is a compact $G$-invariant subset of $D$.

Let $K$ be a compact $G$-invariant subset of $D$; by the paragraph above, such a set $K$ exists. Let $e$ be the identity element of $G$.

Case 1. The function $e$ is a constant, $e(z) \equiv z_{0}$. Then $e=e g=g$ for all $g \in G$. For any $k \in K$, we get $z_{0}=e(k) \in K \subseteq C I R(K)$, hence the lemma holds trivially for this case.

Case 2. The function $e$ is not a constant. Then $e(D)$ is open by the interior mapping theorem [3, p. 92]. For all $w \in D, e(w)=$ $(e \cdot e)(w)=e(e(w))$. So $e(z)=z$ for all $z \in e(D)$, hence for all $z \in D$ by uniqueness of analytic continuation [3, p. 199]. (This part of the proof of Case 2 repeated [13, Lemma 2, p. 704].) Thus $e$ is the identity map on $D$, so $G$ is a group of analytic bijections of $D$, hence a group of Möbius transformations of $D$ by $[4,15.1 .2$, p. 236]. But a Möbius transformation that is analytic on a closed disk $F \subseteq C$ 
maps $F$ onto another closed disk (see [3, Theorem 3.2.1, p. 51]). So if $F \in \mathscr{J}(K)$ and $g \in G$, then $g(F) \in \mathscr{J}(K)$ by $G$-invariance of $K$. Thus $g$ permutes the closed disks $\mathscr{J}(K)$, since $G$ is a group. This yields

$$
\begin{aligned}
g(C I R(K)) & =g(\cap\{F ; F \in \mathscr{J}(K)\})=\cap\{g(F) ; F \in \mathscr{J}(K)\} \\
& =\cap\{F ; F \in \mathscr{J}(K)\}=\operatorname{CIR}(K)
\end{aligned}
$$

for all $g \in G$, hence $\operatorname{CIR}(K)$ is $G$-invariant, and by Lemma 1 (c), also circular hulled.

Let $\mathscr{V}$ be the family of all $G$-invariant circular hulled (hence compact) subsets of $C I R(K)$ ordered downwards by inclusion. The intersection of any linearly ordered subset of of is compact, $G$ invariant, and by Lemma 1(c), circular hulled. By Zorn's lemma, there exists a minimal $P \in \mathscr{Y}$.

Suppose $P$ contains more than one point; we shall show this leads to a contradiction. Let $p, q \in P$ where $p \neq q$. Let $F_{1}, F_{2}$ be the two closed disks of unit radius in $C$ whose boundary circles intersect at both $p$ and $q$. Let $L$ be the lens shaped space $L=$ $F_{1} \cap F_{2}$, and let $F \in \mathcal{J}(P)$. Then $p, q \in P \subseteq F$. Further, the radius $\alpha$ of the closed disk $F$ satisfies $\alpha<1$, since $F \cong D$.

We now need a little plane geometry. If two circles in $C$ of different radii intersect, a major arc of the larger circle cannot lie on or inside the smaller circle. So the portion of the larger circle that lies outside the smaller circle is a major arc. Hence a minor arc of unit radius connecting the two points $p$ and $q$ must lie in the closed disk $F$. Thus $L \subseteq F$.

Since $L \subseteq F$ for all $F \in \mathscr{J}(P)$, we have

$$
L \subseteq \cap\{F ; F \in \mathscr{J}(P)\}=C I R(P)=P,
$$

where the last equality follows from the fact that $P \in \mathscr{Y}$. Let $v=$ $(p+q) / 2$. Then $v \in \operatorname{Int}(L)$, so $v \in \operatorname{Int}(P)$. Therefore $G(v) \subseteq \operatorname{Int}(P)$, since each $g \in G$ is a homeomorphism and $P$ is $G$-invariant. But $G(v)$ is compact and $G$-invariant, so it follows by the first paragraph of Case 2 above that $C I R(G(v))$ is $G$-invariant and circular hulled. Also, $\operatorname{CIR}(G(v)) \leqq C I R(P)=P$. Let $A$ be the smallest closed disk with center at zero such that $P \subseteq A \subseteq D$. Since $G(v) \subseteq \operatorname{Int}(P) \subseteq$ Int $(A)$, the compact $G(v)$ is bounded away from the boundary circle of $A$. Thus there exists $B$, an even smaller closed disk than $A$, such that $B$ has center at zero, and $G(v) \subseteq B \cong \operatorname{Int}(A)$. Clearly, $B \in \mathscr{J}(G(v))$ but $B \notin \mathscr{J}(P)$. Thus $C I R(G(v)) \neq C I R(P)=P$, hence $C I R(G(v))$ is a proper $G$-invariant circular hulled subset of $P$, a contradiction of the minimality of $P \in \mathscr{Y}$, which proves Lemma 2.

We remark that some restriction must be placed on the group 
$G \subseteq H(D)$ to guarantee the existence of a common fixed-point, since the family of all analytic homeomorphisms of $D$ serves as a well known example of a group with no common fixed-points (see [4, Theorem 15.1.2, p. 236]). Although the compactness condition on $G$ cannot be dropped, it can be relaxed. By the use of Lemma 2, it can be shown that a group $G \subseteq H(D)$ has a common fixed-point in $D$ iff $\bar{G} \leqq H(D)$, but we shall not need this in what follows. (Recall that $\bar{G}$ is the closure of $G$ in $E(D)$.)

CoRollary 1. Let $G$ be a finite group of analytic self-maps of $D$. Then $D$ contains a common fixed-point of $G$.

Proof. $G$ is compact.

We suspect that Corollary 1 is known, but we do not know of an explicit reference.

The next result is a complex analogue of a theorem of $\mathrm{T}$. Mitchell [11, Theorem 2, p. 149] concerning common fixed-points of equicontinuous self-maps of the closed unit interval. A semigroup is called left reversible if for every pair of elements $a, b \in S$, there exists a pair $c, d \in S$ such that $a c=b d$.

THEOREM 1. Let $S$ be a left reversible semigroup of analytic self-maps of $D$, and let $K$ be a compact $S$-invariant subset of $D$. Then $C I R(K)$ contains a common fixed-point of $S$.

Proof. By [4, Theorem 15.2.3, p. 246], $S$ is a normal family of analytic maps so $\bar{S}$ is a compact subset of $E(D)$. Each $s \in S$ maps $D$ into $D$, so each $t \in \bar{S}$ maps $D$ into $\bar{D}$, the closure of $D$ in $C$.

Suppose that $\bar{S} \nsubseteq H(D)$; then there exists $t_{0} \in \bar{S}$ for which $t_{0}(D) \nsubseteq D$. But each nonconstant function $t \in \bar{S}$ maps $D$ into $D$ by the interior mapping theorem [3, p. 92]. Hence $t_{0}(z) \equiv z_{0}$, where $z_{0} \notin D$, for all $z \in D$. There exists a sequence $s_{n} \in S$ such that $s_{n} \rightarrow t_{0}$ in the topology of $E(D)$. Thus for each $k \in K, s_{n}(k) \rightarrow z_{0}$, a contradiction, since $s_{n}(k) \in K$ and $z_{0} \notin K$. Hence $\bar{S} \subseteq H(D)$, so $\bar{S}$ is a compact topological semigroup.

We now repeat an argument used in the proof of [11, Theorem 1, p. 147]. Let $a, b \in \bar{S}$, then there exist sequences $a_{m}$ and $b_{m}$ in $S$ such that $a_{m} \rightarrow a$ and $b_{m} \rightarrow b$. By left reversibility of $S$, there exist sequences $c_{m}$ and $d_{m}$ in $S$ such that $a_{m} c_{m}=b_{m} d_{m}$. But $\bar{S}$ is compact, so there exist subsequences $c_{n}$ and $d_{n}$ for which $c_{n} \rightarrow c$ and $d_{n} \rightarrow d$ for some $c, d \in \bar{S}$. Hence

$$
\begin{aligned}
a c & =\left(\lim a_{n}\right)\left(\lim c_{n}\right)=\lim \left(a_{n} c_{n}\right) \\
& =\lim \left(b_{n} d_{n}\right)=\left(\lim b_{n}\right)\left(\lim d_{n}\right)=b d,
\end{aligned}
$$


where the second and fourth equalities hold by virtue of the joint continuity of the product in $\bar{S}$. Thus $\bar{S}$ is left reversible.

An induction argument shows that if $\left\{a_{1}, a_{2}, \cdots, a_{r}\right\}$ is any finite subset of $\bar{S}$, there exists a finite subset $\left\{b_{1}, b_{2}, \cdots, b_{r}\right\}$ of $\bar{S}$ such that $a_{1} b_{1}=a_{2} b_{2}=\cdots=a_{r} b_{r}$. Hence

$$
\cap\left\{a_{j} \bar{S} ; j=1, \cdots, r\right\} \supseteqq \cap\left\{a_{j} b_{j} ; j=1, \cdots, r\right\}=\left\{a_{1} b_{1}\right\} \neq \varnothing .
$$

Thus the family $\{a \bar{S} ; a \in \bar{S}\}$ of compact, hence closed, subsets of $\bar{S}$ has the finite intersection property, so $\cap\{a \bar{S} ; a \in \bar{S}\} \neq \varnothing$ by compactness of $\bar{S}$. But each right ideal of $\bar{S}$ contains some principal right ideal $a \bar{S}$; and the intersection of a family of right ideals of $\bar{S}$ is, if nonempty, also a right ideal of $\bar{S}$. Therefore $\cap\{a \bar{S} ; a \in \bar{S}\}$ is the unique minimal right ideal of $\bar{S}$. By [5, Theorem 1, p. 57], it follows that $\bar{S}$ contains a compact group $G$ for which $\bar{S} G \leqq G$. In particular, if $e$ is the identity element of $G$, then $s e \in G$ for all $s \in S$.

For any $g \in G$, there exists a sequence $s_{n} \in S$ such that $s_{n} \rightarrow g$. But for each $k \in K, s_{n}(k) \in K$, therefore $g(k)=\lim \left(s_{n}(k)\right) \in K$. Hence $K$ is $G$-invariant, so $C I R(K)$ contains a common fixed-point $p$ of $G$ by Lemma 2. Thus for any $s \in S$, we have $s(p)=s(e(p))=(s e)(p)=p$, which proves Theorem 1.

CoROLlary 2. Let $f$ be an analytic self-map of $D$, and let $K$ be a compact $f$-invariant subset of $D$. Then $C I R(K)$ contain a fixedpoint of $f$.

Proof. The semigroup $S$ generated by $f$ is Abelian, hence left reversible.

We note that the fixed-point whose existence is asserted in Theorem 1 is unique if $S$ contains an $s$ which is not the identity map on $D$. For in such a case, if $s$ is an analytic homeomorphism of $D$ onto $D$, and $D$ contains a fixed-point of $s$, then the fixed-point in $D$ is unique by [13, Lemma $5, \mathrm{p} .705]$. On the other hand, if $s$ is an analytic self-map of $D$, but not a homeomorphism onto $D$, then there exists $z_{0} \in \bar{D}$ such that $s^{n}(z) \rightarrow z_{0}$ for all $z \in D$ by [13, p. 705]. So if $s$ has a fixed-point $p \in D$, then we have $p=s^{n}(p) \rightarrow z_{0}$, thus $p=z_{0}$, hence $p$ is unique.

If in Theorem 1, we drop the assumption that there exists an $S$-invariant compact subset $K \subseteq D$, then $D$ need not contain a common fixed-point of $S$. Even if one also adds the requirement that each $s \in S$ is a continuous self-map of $\bar{D}$ and is analytic on $D$, a left reversible semigroup $S$ need not have a common fixed-point in $\bar{D}$. The group of all analytic bijections of $D$, extended continuously to $\bar{D}$, serves as an easy counterexample. 
Similarly, if we let $K=\{-1 / 2,1 / 2\}, s_{1}(z)=-1 / 2$, and $s_{2}(z)=1 / 2$ for all $z \in D$, then the semigroup $S=\left\{s_{1}, s_{2}\right\}$ has no common fixedpoint in $D$, let alone in $C I R(K)$, which shows that one cannot entirely drop the assumption in Theorem 1 that $S$ is left reversible.

4. Actions of semitopological semigroup. A. T.-M. Lau [9, Theorems 3.2, 4.1], [10, p. 381] has shown that three fixed-point theorems that were known to hold for left reversible semigroups could be extended to the case of actions of semitopological semigroups, $S$, for which the space $A P(S)$, the space of almost periodic functions on $S$, has a left invariant mean. (In [9], Lau also obtained converses to the extended forms of two of these theorems.) Lau's work suggests to us that Theorem 1 may also be generalizable to actions of such semitopological semigroups, which in fact, it is. We need first some terminology.

A semitopological semigroup is a semigroup with a Hausdorff topology in which the product st is separately continuous. If $T$ is a semitopological semigroup, $C B(T)$ will denote the space of continuous bounded real-valued functions on $T$, where $C B(T)$ is given the supremum norm. A function $h \in C B(T)$ is almost periodic if $\left\{\iota_{a} h ; a \in T\right\}$ is relatively compact in $C B(T)$, where $\left(\ell_{a} h\right)(s)=h(a s)$ for all $a, s \in T$. Then $A P(T)$, the space of all almost periodic functions $h \in C B(T)$, is a norm closed linear subspace of $C B(T)$ which contains the constant functions, and which satisfies $\iota_{s}(A P(T)) \subseteq A P(T)$ for all $s \in T$ (see $[2$, p. 80]).

Let $W$ be any norm closed linear subspace of $C B(T)$ that contains the constant functions, and satisfies $\ell_{s}(W) \subseteq W$ for all $s \in T$. A mean on $W$ is an element $\mu \in W^{*}$, the continuous dual of $W$, for which $\|\mu\|=1$ and $\mu(h) \geqq 0$ whenever $h \in W$ and $h \geqq 0$. If a mean $\mu$ on $W$ satisfies $\mu\left(l_{s} h\right)=\mu(h)$ for all $s \in T$ and all $h \in W$, then $\mu$ is a LIM (left invariant mean) on $W$.

An action of a topological semigroup $T$ on $D$ is a map $\theta: T \times$ $D \rightarrow D$ for which $\theta\left(t_{1} t_{2}, z\right)=\theta\left(t_{1}, \theta\left(t_{2}, z\right)\right)$ for all $t_{1}, t_{2} \in T$ and all $z \in D$. The action is separately continuous if $\theta$ is continuous in each variable when the other variable is held fixed. For an action $T \times D \rightarrow D$, define a map $\lambda: T \rightarrow D^{D}$ by $(\lambda t)(z)=\theta(t, z)$ for $t \in T, z \in D$. The action is analytic if $\lambda: T \rightarrow H(D)$, a subset $K \subseteq D$ is $T$-invariant if $(\lambda T) K \subseteq K$, and an element $p \in D$ is a common fixed-point of $T$ if $(\lambda T)(p)=\{p\}$.

The extension from Theorem 1 to Theorem 2 below is based upon the known result, implicit in the work of Deleeuw and Glicksberg in [2], that if there exists a continuous homomorphism from a semitopological semigroup $T$ onto a dense subset of a compact 
topological semigroup $S$, and if $A P(T)$ has a LIM, then $S$ is left reversible. This result is obtained by collecting four items from [2]; these items are explicitly cited in the proof below.

THEOREM 2. Let $T \times D \rightarrow D$ be a separately continuous analytic action of a semitopological semigroup $T$ on $D$, and let $K$ be a compact T-invariant subset of $D$. If $A P(T)$ has a LIM, then $\operatorname{CIR}(K)$ contains a common fixed-point of $T$.

Proof. For $t_{1}, t_{2} \in T$, we have

$$
\begin{aligned}
\left(\lambda\left(t_{1} t_{2}\right)\right)(z) & =\theta\left(t_{1} t_{2}, z\right)=\theta\left(t_{1}, \theta\left(t_{2}, z\right)\right)=\left(\lambda t_{1}\right)\left(\theta\left(t_{2}, z\right)\right) \\
& =\left(\lambda t_{1}\right)\left(\left(\lambda t_{2}\right)(z)\right)=\left(\left(\lambda t_{1}\right)\left(\lambda t_{2}\right)\right)(z)
\end{aligned}
$$

for all $z \in D$. Thus $\lambda\left(t_{1} t_{2}\right)=\left(\lambda t_{1}\right)\left(\lambda t_{2}\right)$, so $\lambda$ is a homomorphism of $T$ into $H(D)$.

Let $t \in T$, and let $\left\{t_{r}\right\}$ be a net in $T$ for which $t_{r} \rightarrow t$. Then for all $z \in D$,

$$
\lim \left(\left(\lambda t_{\gamma}\right)(z)\right)=\lim \left(\theta\left(t_{\gamma}, z\right)\right)=\theta(t, z)=(\lambda t)(z),
$$

thus $\lambda t_{r}$ converges pointwise on elements of $D$ to $\lambda t$. In order to show that the homomorphism $\lambda: T \rightarrow H(D)$ is continuous, we must show that $\lambda t_{\gamma}$ converges uniformly on compact subsets of $D$ to $\lambda t$. Denote $\lambda T$ by $S$, then a repetition of the argument used in the first two paragraphs of the proof of Theorem 1 yields that $\bar{S}$ is a compact topological semigroup. On $H(D)$, the topology of pointwise convergence on elements of $D$ is Hausdorff and is weaker than the topology of uniform convergence on compact subsets of $D$. But $\bar{S}$ is compact in the second topology, so the two topologies coincide on $\bar{S}$ by [8, Theorem 8, p. 141], hence $\lambda$ is a continuous homomorphism of $T$ onto $S$, a dense subset of the compact topological semigroup $\bar{S}$.

Let $k \in K$, then $S k \subseteq K$ by hypothesis. Since the map $\bar{S} \rightarrow K$, given by $s \rightarrow s k$, is continuous, we get that the set $Z=\{s \in \bar{S}$; sk $\in K\}$ is a closed subset of $\bar{S}$ that contains $S$, so $Z=\bar{S}$, thus $K$ is $\bar{S}$ invariant. But $A P(T)$ has a LIM, hence so does $C B(\bar{S})$, by [2, Lemma 5.2, p. 81] and [2, Lemma 2.10, p. 71], therefore the compact topological semigroup $\bar{S}$ is left reversible by [2, Corollary 2.4, p. 67] and [2, Lemma 2.8, p. 70]. It follows by Theorem 1 that $\bar{S}$, hence $S$, has a common fixed-point in $C I R(K)$, which proves Theorem 2.

It is known (see Holmes and Lau [6, Corollary 1, p. 333] and Lau [9, Theorem 4.1, p. 74]) that every left reversible semitopological semigroup $T$ has the property that $A P(T)$ has a LIM. We also 
remark that there exist semitopological semigroups $T$ which are not left reversible but for which $A P(T)$ has a LIM (see [6, p. 335]), hence Theorem 2 is a proper generalization of Theorem 1.

\section{REFERENCES}

1. M. M. Day, Fixed point theorems for compact convex sets, Illinois J. Math., 5 (1961), $585-590$.

2. K. DeLeeuw and I. Glicksberg, Applications of almost period compactifications, Acta Math., 105 (1961), 63-97.

3. E. Hille, Analytic Function Theory, Vol. I, Ginn, Boston, Ma., 1959.

4. —, Analytic Function Theory, Vol. II, Ginn, Boston, Ma., 1962.

5. K. H. Hofman and P. S. Mostert, Elements of Compact Semigroups, Merrill, Columbus, Ohio, 1966.

6. R. D. Holmes and A. T. Lau, Non-expansive actions of topological semigroups and fixed points, J. London Math. Soc., (2), 5 (1972), 330-336.

7. H. D. Junghenn, Some general results on fixed-points and invariant means, Semigroup Forum, 11 (1975), 153-164.

8. J. L. Kelley, General Topology, Van Nostrand, Princeton, N. J., 1955.

9. A. T.-M. Lau, Invariant means on almost periodic functions and fixed point properties, Rocky Mountain J. Math., 3 (1973), 69-76.

10. Invariant means on almost periodic functions and equicontinuous actions, Proc. Amer. Math. Soc., 49 (1975), 379-382.

11. T. Mitchell, Common fixed-points for equicontinuous semigroups of mappings, Proc. Amer. Math. Soc., 33 (1972), 146-150.

12. R. Oliver, Fixed-point sets of group actions on finite acyclic complexes, Comment. Math. Helvetici, 50 (1975), 155-177.

13. A. L. Shields, On fixed points of commuting analytic functions, Proc. Amer. Math. Soc., 15 (1964), 703-706.

14. J. C. S. Wong, Topological semigroups and representations, Trans. Amer. Math. Soc., 200 (1974), 89-109.

Received September 21, 1978.

Temple University

Philadelphia, PA 19122 



\section{PACIFIC JOURNAL OF MATHEMATICS}

\section{EDITORS}

DONALD BABBITT (Managing Editor)

University of California

Los Angeles, California 90024

HUgo RossI

University of Utah

Salt Lake City, UT 84112

C. C. MOORE and ANDREW OGG

University of California

Berkeley, CA 94720

\section{J. DUGUNDJI}

Department of Mathematics University of Southern California Los Angeles, California 90007

R. Finn and J. Milgram Stanford University Stanford, California 94305

\section{ASSOCIATE EDITORS}

E. F. BECKENBACH

B. H. NEUMANN

F. WOLF

K. YoshidA

\section{SUPPORTING INSTITUTIONS}

UNIVERSITY OF BRITISH COLUMBIA CALIFORNIA INSTITUTE OF TECHNOLOGY UNIVERSITY OF CALIFORNIA MONTANA STATE UNIVERSITY UNIVERSITY OF NEVADA, RENO NEW MEXICO STATE UNIVERSITY OREGON STATE UNIVERSITY UNIVERSITY OF OREGON
UNIVERSITY OF SOUTHERN CALIFORNIA STANFORD UNIVERSITY UNIVERSITY OF HAWAII UNIVERSITY OF TOKYO UNIVERSITY OF UTAH WASHINGTON STATE UNIVERSITY UNIVERSITY OF WASHINGTON 


\section{Pacific Journal of Mathematics}

Vol. 85, No. $1 \quad$ September, 1979

Ralph Alexander, Metric averaging in Euclidean and Hilbert spaces...... 1

B. Aupetit, Une généralisation du théorème de Gleason-Kahane-Żelazko

pour les algèbres de Banach..............................

Lung O. Chung, Jiang Luh and Anthony N. Richoux, Derivations and

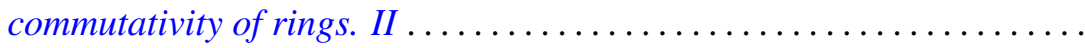

Lynn Harry Erbe, Integral comparison theorems for third order linear

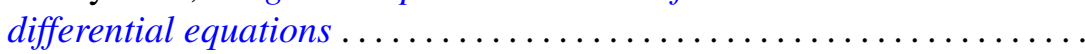

Robert William Gilmer, Jr. and Raymond Heitmann, The group of units of a

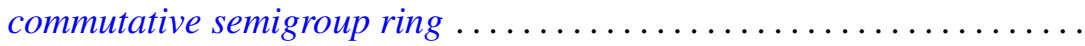

George Grätzer, Craig Robert Platt and George William Sands, Embedding lattices into lattices of ideals ...........................

Raymond D. Holmes and Anthony Charles Thompson, $n$-dimensional area and content in Minkowski spaces ....................... 77

Harvey Bayard Keynes and M. Sears, Modelling expansion in real flows....

Taw Pin Lim, Some classes of rings with involution satisfying the standard

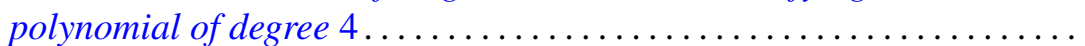

Garr S. Lystad and Albert Robert Stralka, Semilattices having bialgebraic congruence lattices ................................... 131

Theodore Mitchell, Invariant means and analytic actions . . . . . . . . . 145

Daniel M. Oberlin, Translation-invariant operators of weak type ........ 155

Raymond Moos Redheffer and Wolfgang V. Walter, Inequalities involving

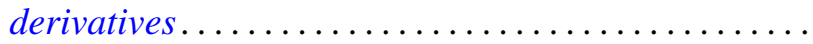

Eric Schechter, Stability conditions for nonlinear products and semigroups ................................

Jan Søreng, Symmetric shift registers ........................ 201

Toshiji Terada, On spaces whose Stone-Čech compactification is $\mathrm{Oz} \ldots \ldots .231$

Richard Vrem, Harmonic analysis on compact hypergroups ... 